\title{
Article
}

\section{ENERGETIC PARTICLE DIFFUSION IN CRITICALLY BALANCED TURBULENCE}

Laitinen, Timo Lauri Mikael, Dalla, Silvia, Kelly, J. and Marsh, Michael

Available at http://clok.uclan.ac.uk/7322/

Laitinen, Timo Lauri Mikael ORCID: 0000-0002-7719-7783, Dalla, Silvia ORCID: 0000-0002-7837-5780, Kelly, J. and Marsh, Michael (2013) ENERGETIC PARTICLE DIFFUSION IN CRITICALLY BALANCED TURBULENCE. The Astrophysical Journal, 764 (2). pp. 168-176. ISSN 0004-637X

It is advisable to refer to the publisher's version if you intend to cite from the work. http://dx.doi.org/10.1088/0004-637x/764/2/168

For more information about UCLan's research in this area go to http://www.uclan.ac.uk/researchgroups/ and search for <name of research Group>.

For information about Research generally at UCLan please go to http://www.uclan.ac.uk/research/

All outputs in CLoK are protected by Intellectual Property Rights law, including Copyright law. Copyright, IPR and Moral Rights for the works on this site are retained by the individual authors and/or other copyright owners. Terms and conditions for use of this material are defined in the policies page.

\section{CLoK}

Central Lancashire online Knowledge www.clok.uclan.ac.uk

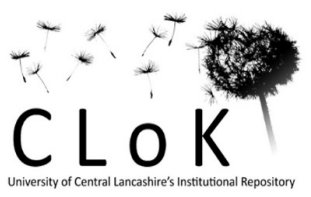




\title{
ENERGETIC PARTICLE DIFFUSION IN CRITICALLY BALANCED TURBULENCE
}

\author{
T. Laitinen, S. Dalla, J. Kelly, and M. Marsh \\ Jeremiah Horrocks Institute, University of Central Lancashire, PR1 2HE Preston, UK \\ Received 2012 October 23; accepted 2012 December 23; published 2013 February 4
}

\begin{abstract}
Observations and modeling suggest that the fluctuations in magnetized plasmas exhibit scale-dependent anisotropy, with more energy in the fluctuations perpendicular to the mean magnetic field than in the parallel fluctuations and the anisotropy increasing at smaller scales. The scale dependence of the anisotropy has not been studied in full-orbit simulations of particle transport in turbulent plasmas so far. In this paper, we construct a model of critically balanced turbulence, as suggested by Goldreich \& Sridhar, and calculate energetic particle spatial diffusion coefficients using full-orbit simulations. The model uses an enveloped turbulence approach, where each two-dimensional wave mode with wavenumber $k_{\perp}$ is packed into envelopes of length $L$ following the critical balance condition, $L \propto k_{\perp}^{-2 / 3}$, with the wave mode parameters changing between envelopes. Using full-orbit particle simulations, we find that both the parallel and perpendicular diffusion coefficients increase by a factor of two, compared to previous models with scale-independent anisotropy.
\end{abstract}

Key words: cosmic rays - diffusion - turbulence

Online-only material: color figures

\section{INTRODUCTION}

The origin of solar energetic particles (SEPs) is one of the unsolved problems in heliospheric physics. Both flares and coronal mass ejections are capable of accelerating particles, and studies analyzing SEP events give differing conclusions on the main particle accelerators (see, e.g., Cane et al. 2010; Gopalswamy et al. 2012; Aschwanden 2012). The interpretation of SEP events is complicated due to the particle propagation effects caused by the turbulent interplanetary magnetic field. SEP events are observed at a large range of latitudes and longitudes (e.g., Dalla et al. 2003; Liu et al. 2011; Dresing et al. 2012), suggesting possible cross-field transport of the SEPs. Thus, in order to understand the SEP acceleration in the solar eruptions, we must first understand the nature of SEP transport in the heliosphere.

Cosmic-ray research is typically based on two theoretical approaches: the diffusion-convection equation (Parker 1965) and the quasilinear approach (QLT; Jokipii 1966). The original description of the cross-field transport of a charged particle as propagation in random-walking magnetic field lines (Jokipii 1966) has been improved to take into account the interplay between parallel and perpendicular propagation effects (Matthaeus et al. 2003; Shalchi 2010). The spread of the particles observed in the SEP events, however, remains difficult to explain, as they require larger ratios of the perpendicular-to-parallel diffusion coefficients than what can be expected from the theoretical approaches (Dresing et al. 2012).

Recently, particle transport research has benefited from numerical simulations utilizing particle full-orbit simulations, which have the advantage of needing no a priori assumptions for the particle propagation. Beresnyak et al. (2011) and Wisniewski et al. (2012) have recently studied particle propagation using MHD-simulated turbulence. However, as MHD simulations are limited to a small range of scales, most full-orbit particle simulations describe the fluctuating fields as a superposition of Fourier modes on a constant magnetic field (e.g., Giacalone \& Jokipii 1999; Qin 2002; Qin et al. 2002; Zimbardo et al. 2006; Ruffolo et al. 2008).
Observations and modeling suggest that the fluctuations in magnetized plasmas are anisotropic, with more energy in the fluctuations perpendicular to the mean magnetic field than in the parallel fluctuations (e.g., Shebalin et al. 1983; Bieber et al. 1996). The anisotropy is also scale dependent, with structures at smaller scales being more anisotropic than the larger scales. The scale dependence of the anisotropy was predicted by Goldreich \& Sridhar (1995, hereafter GS95), who noted that as the turbulence amplitudes increase at small scales, they introduce variation along the mean field direction. This is due to the fact that when interacting, the wave packets propagate through each other, along the mean magnetic field. When the changes in the interacting wave packets reach nonlinear amplitudes, both of the interacting waves change, and for this reason also the outcome of the interaction changes. This takes place on nonlinear timescales $\tau_{\mathrm{NL}}$, when the wave packet's front has propagated a distance $\propto V_{A} \tau_{\mathrm{NL}}$, where $V_{A}$ is the Alfvén velocity. Using the nonlinear timescale from Kolmogorov scaling, GS95 obtained a critical balance, $k_{\|} \propto k_{\perp}^{2 / 3}$, between the parallel and perpendicular wavenumbers. The critical balance between structure scales has been found in MHD turbulence simulations (e.g., Cho et al. 2002) and observed in the solar wind turbulence (e.g., Horbury et al. 2008; Podesta 2009). In order to understand the propagation of particles in heliospheric plasmas, the scale dependence of the turbulence anisotropy should thus be taken into account.

In this work, we study the particle propagation in the critically balanced turbulence of GS95. To model the turbulence, we use the enveloping approach of Laitinen et al. (2012). The enveloping approach improves the earlier models that use infinite, linear plane waves by breaking the phase coherence of the waves in the direction of the mean magnetic field. Such a loss of coherence is expected in the plasma in the heliosphere, as the waves evolve in a nonlinear manner (see, e.g., Tu \& Marsch 1995, for a review). Laitinen et al. (2012) studied the effect of structures on the transport and used a fixed envelope length of the order of turbulence correlation length. In the present paper, we study the particle propagation in turbulence with scaledependent anisotropy, and the enveloping is done separately for 


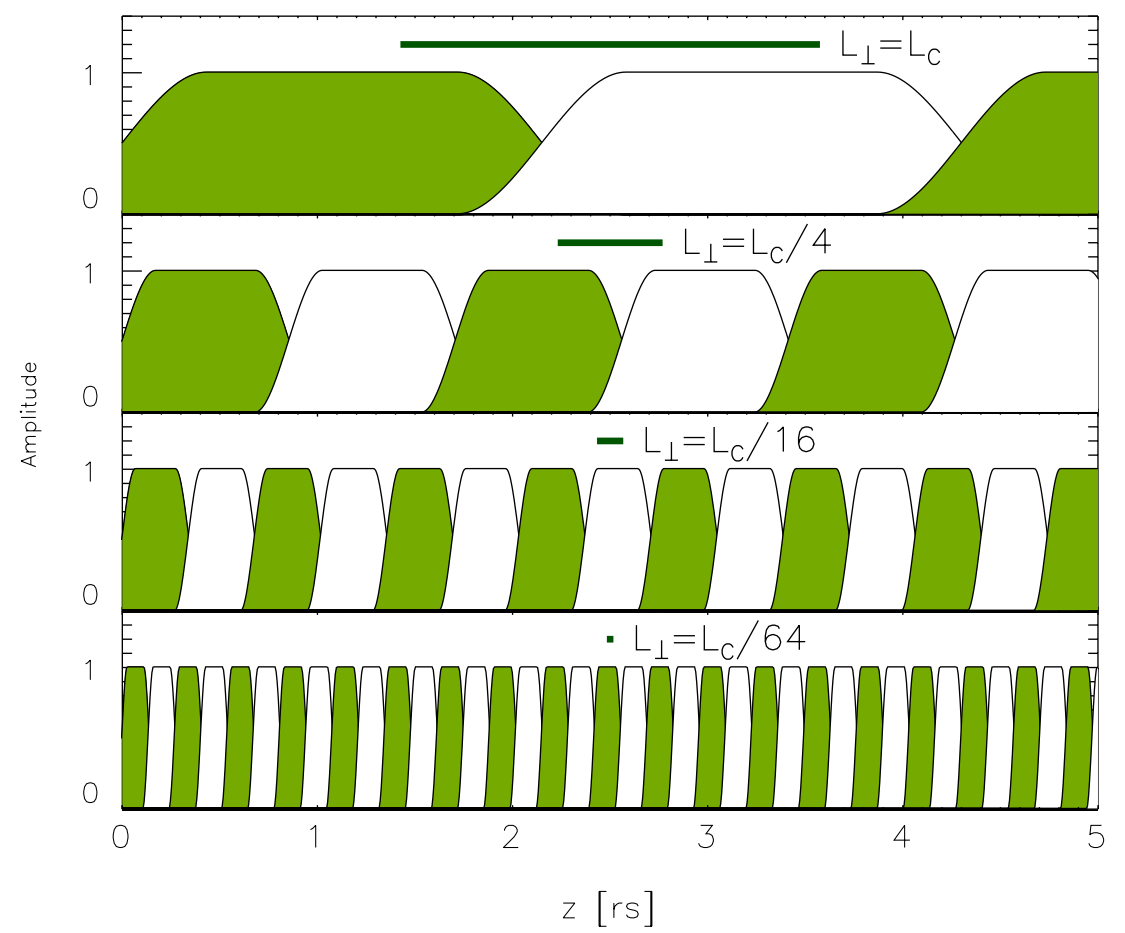

Figure 1. Green and white packets represent the envelopes along the mean field direction, for four different perpendicular scales, shown by the thick horizontal lines. The steepness parameter is $\sigma=10$ in this figure.

(A color version of this figure is available in the online journal.)

each wave mode, with the envelope length following the critical balance scaling, $L \propto k_{\perp}^{-2 / 3}$, where $L$ is the envelope length and $k_{\perp}$ is the wavenumber of the enveloped Fourier mode. The Fourier modes are chosen to be two-dimensional modes, with their wave vector perpendicular to the mean magnetic field. Thus, the variation of the magnetic field along the mean field is purely due to the enveloping of the modes. We use particle fullorbit simulations in the modeled turbulence and calculate the diffusion coefficients for energetic particles, comparing them to results given in previous work. In Section 2, we describe the critically balanced turbulence model and the method used for obtaining the energetic particle diffusion coefficients. In Section 3, we study the scale dependence of the turbulence in the developed model and report changes in the energetic particle diffusion coefficients, compared to the traditional composite model. We discuss the results and draw our conclusions in Section 4.

\section{MODEL}

\subsection{Turbulence Model}

In our model, the magnetic field consists of a uniform and constant background field, $B_{0}$, overlaid with a turbulent field, $\delta \mathbf{B}(x, y, z)$, with the total magnetic field given as

$$
\mathbf{B}=\mathbf{B}_{0} \hat{e}_{z}+\delta \mathbf{B}(x, y, z) .
$$

For the background field, we use $B_{0}=5 \mathrm{nT}$, consistent with the magnetic field magnitude at $1 \mathrm{AU}$.

We model the scale-dependent turbulence by using the turbulence envelope approach, introduced by Laitinen et al. (2012). In the approach, the turbulence is enveloped into packets along the mean field direction (see Figure 1). From one envelope to the next, the parameters of the wave modes (random phase, wave vector direction) change. Thus, a particle propagating from an envelope to another interacts with a coherent Fourier mode only within one envelope, and the linear coherence is broken when it enters a different envelope.

The scale dependence of the turbulence in our model is achieved through the selection of the envelope lengths, which in the study of Laitinen et al. (2012) were constant. Here, the enveloping is done separately for each wave mode, with the wave scale $\lambda_{\perp n}=2 \pi / k_{\perp n}$, where $k_{\perp n}$ is the wavenumber of the two-dimensional mode. The envelope length, $L_{n}$, follows the critical balance scaling, $L_{n}=L_{C}^{1 / 3} \lambda_{\perp n}^{2 / 3}$, where $L_{c}$ is the scale where parallel and perpendicular scales are in balance. As a result, for wave scales $\lambda_{\perp n}<L_{C}$ the perpendicular wave scales decrease faster than the parallel envelope scales, resulting in scale-dependent anisotropy. This process is depicted in Figure 1, where we show the wave scale (thick horizontal line) compared to the envelope length scales, for four different wave scales.

The amplitude of mode $n$ for envelope $i$ is modulated by the function

$$
\mathfrak{A}_{n, i}(z)=\frac{1}{2}\left[F\left\{\frac{z-z_{i}}{2 S_{n}}\right\}-F\left\{\frac{z-L_{n}-z_{i}}{2 S_{n}}\right\}\right],
$$

where $z_{i}$ marks the beginning of the envelope and $z$ is the distance along the direction of the constant magnetic field. The profile of the envelope is given by the function

$$
F\{z\}= \begin{cases}-1 & z<-1 \\ \frac{3}{2} z-\frac{1}{2} z^{3} & -1 \leqslant z \leqslant 1 \\ 1 & z>1 .\end{cases}
$$

This approximates the often-used tanh profile, which enables analyzing differentiable profiles that can have steep edges, approaching a step-function shape. The polynomial description 


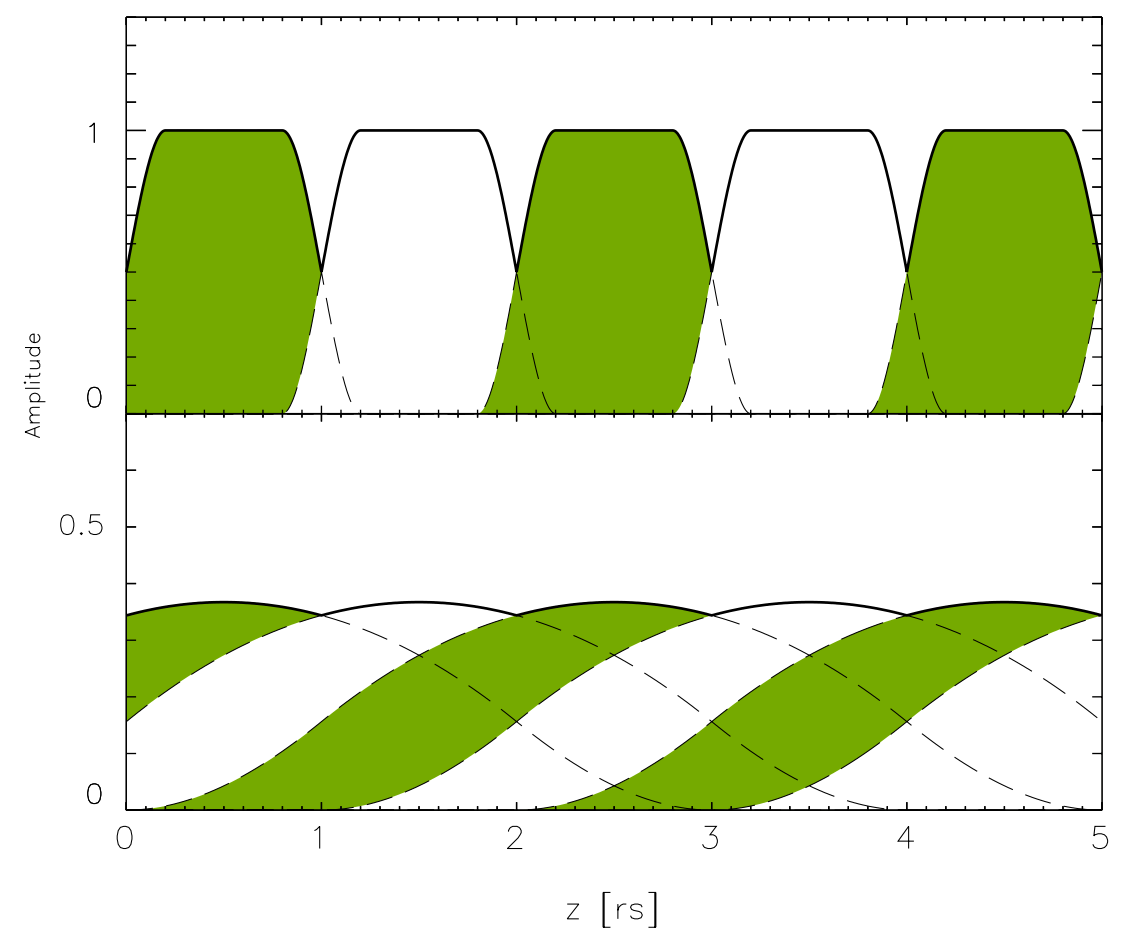

Figure 2. Green and white packets represent the envelopes along the mean field direction, for $\sigma=10$ (top panel) and $\sigma=1$ (bottom panel). The envelope length $L_{n, i}=1$ for these profiles.

(A color version of this figure is available in the online journal.)

was chosen for numerical efficiency. The steepness of the envelope profile is given by the parameter $S_{n}=L_{n} / \sigma$, and $\sigma$ is a dimensionless parameter relating the envelope length to the rise profile length.

In the modeling, we consider that a wave mode with a given wave vector direction and random phase gives its energy to another mode with the same modulus of $k_{n}$, without losses, thus keeping the sum of the amplitudes of the modes constant, $\sum_{i} \mathfrak{A}_{n, i}(z)=1$. This is achieved by selecting $z_{i+1}=z_{i}+L_{n}$.

The steepness of the envelope is related to the transfer rate of energy from one mode to another, due to the critical balancing of the turbulence. The rate of change depends on the relative wavenumber directions of the interacting modes (e.g., Luo \& Melrose 2006), which results in some interactions transferring the energy faster than others. In our model, however, we describe phenomenologically only the result of the critically balanced 3wave interactions, and thus an accurate description of the rise profile is not possible on this level. Instead, we consider in this study two different profiles, a gradual change with $\sigma=1$ and a rapid change with $\sigma=10$. As shown in Figure 2, for $\sigma=10$, the energy is transferred from one mode to another in an intermittent manner, whereas for $\sigma=1$, the interaction is more gradual between multiple modes. The distance along the mean field direction over which one mode dominates over the others remains the same, the envelope length, $L_{n}$, for both values of $\sigma$.

The turbulence field is given as a sum of $M_{n}$ envelopes and $N$ Fourier modes, as

$$
\delta \mathbf{B}(x, y, z)=\sum_{n=1}^{N} \sum_{i=1}^{M_{n}} \mathfrak{A}_{n, i}(z) A\left(k_{n}\right) \hat{\xi}_{n, i} e^{i\left(k_{\perp n} z_{n, i}^{\prime}+\beta_{n, i}\right)}
$$

In this study, the Fourier modes in the envelopes are twodimensional modes, with the wave vector $\mathbf{k}_{\perp n}$ perpendicular to the mean magnetic field. Thus, the variation on parallel scales in the model is due to the enveloping only. The polarization vector $\hat{\xi}_{n, i}$ lies in the $x y$-plane and is perpendicular to both the mean magnetic field and $\mathbf{k}_{n}$, in order to satisfy $\nabla \cdot \mathbf{B}=0$. Thus,

$$
\hat{\xi}_{n, i}=i \hat{\mathbf{y}}_{n}^{\prime},
$$

where the coordinate system $\mathbf{r}^{\prime}$ is obtained from $\mathbf{r}$ with a rotation matrix (Giacalone \& Jokipii 1999). The wave propagation directions in the $x y$-plane, as well as the random phases $\beta_{n, i}$, are chosen from a uniform random distribution.

The fluctuation amplitude $A\left(k_{n}\right)$ is given by a power-law spectrum

$$
A^{2}\left(k_{n}\right)=B_{1}^{2} \frac{G_{n}}{\sum_{n=1}^{N} G_{n}}, \quad G\left(k_{n}\right)=\frac{\Delta V_{n}}{1+\left(k_{n} L_{c}\right)^{\gamma}},
$$

where $B_{1}^{2}$ is the variance of the magnetic field, $L_{c}$ is the spectrum's turnover scale, $\gamma$ is the spectral index, and $\Delta V_{n}$ specifies the volume element in $k$-space that the discrete mode $k_{\perp n}$ represents. For the turnover scale, we use $L_{c}=2.15 r_{\odot}$, where $r_{\odot}$ is the solar radius. For two-dimensional turbulence, the spectral index is $8 / 3$ and the factor $\Delta V_{n}=2 \pi k_{n} \Delta k_{n}$. In the simulations, we use logarithmically spaced wave modes with the wavenumber running from $2 \pi / 1 \mathrm{AU}$ to $2 \pi / 10^{-4} \mathrm{AU}$.

To compare the turbulent fields and their effects on SEP propagation, we scale the fluctuation amplitudes so that the average energy density in the fluctuating field is independent of the enveloping parameters. The scaling factor is obtained through numerical integration.

\subsection{Energetic Particle Simulations}

We study particle propagation in the modeled turbulent magnetic fields by integrating the fully relativistic equation 
of motion of energetic protons using the simulation code by Dalla \& Browning (2005). The code uses the Bulirsh-Stoer method (Press et al. 1993), with adaptive timestepping to control the accuracy by limiting the error between consecutive steps to a given tolerance. We simulate 2048 particles in 10 field realizations, with different wave mode random phases and wave vector directions in each realization, thus giving a total of 20,480 particles. Each realization has $N=128$ Fourier modes, whereas the number of envelopes depends on the energy of the particle. The particles are simulated for $\sim 100$ parallel diffusion times. The particle diffusion coefficient is obtained with

$$
\kappa_{\zeta \zeta}=\frac{\left\langle\Delta \zeta^{2}\right\rangle}{2 t}, \quad \zeta=x, y, z
$$

(e.g., Giacalone \& Jokipii 1999), with $\kappa_{\|}$equal to $\kappa_{z z}$, whereas $\kappa_{\perp}$ is obtained as the mean of $\kappa_{x x}$ and $\kappa_{y y}$. For further details of the simulation scheme and verification against the results of Giacalone \& Jokipii (1999), see Laitinen et al. (2012).

\section{RESULTS}

\subsection{Scale dependence of the Turbulence}

The goal of this work is to study particle motion in a turbulent field that has a scale-dependent anisotropy that corresponds to the critically balanced scaling suggested by GS95. As the first step, we compare the composite turbulence model of Giacalone \& Jokipii (1999) to the one presented in this study. We do this by calculating the two-point correlation function,

$$
C_{x x}(\mathbf{s})=\left\langle\delta B_{x}(\mathbf{r}) \delta B_{x}(\mathbf{r}+\mathbf{s})\right\rangle,
$$

where $\mathbf{s}$ is the scale vector that the correlation is calculated for and the angle brackets denote ensemble average. We calculate the correlation function as a function of $s_{\perp}$ and $s_{\|}$, that is, across and along the mean field direction. The correlation function is calculated from the dissipation scales to the turnover scale $L_{c}$ of the spectrum and presented in Figure 3, where the contours are selected to represent correlations at equidistant scales, $s$, rather than values of the correlation function, $C_{x x}$. The correlation contours for the GS95 model are ellipses (appearing rectangular in the log-log presentation of Figure 3), with the aspect ratio increasing at smaller scales. A similar trend cannot be seen in the composite model.

To quantify the scale dependence of the anisotropy, we calculate the axis ratio of the contours. This is done by finding values of the parallel and perpendicular scales, $s_{\|}$and $s_{\perp}$, that satisfy $C_{x x}\left(s_{\perp}, 0\right)=C_{x x}\left(0, s_{\|}\right)$. We present the axis ratio $s_{\|} / s_{\perp}$ as a function of $s_{\perp}$, for the turbulence models, in Figure 4. The dash-dotted straight line represents the GS95 scaling, the solid and the dotted curves (green and red in the online version) represent the phenomenological model of this work, with two different values of the steepness parameter $\sigma$, and the dashed curve (blue in the online version) represents the composite model of Giacalone \& Jokipii (1999). As can be seen, the axis ratio in the model presented in this work is clearly scale dependent and follows the trend of the GS95 scaling. There is a significant dependence of the level of anisotropy on the steepness parameter, with the steep profile for the energy change between the wave modes, represented by $\sigma=10$, producing values closer to isotropy when the axis scales approach the isotropy scale $L_{c}$.

At large scales, the phenomenological model deviates from the GS95 model. This is due to the different form of the
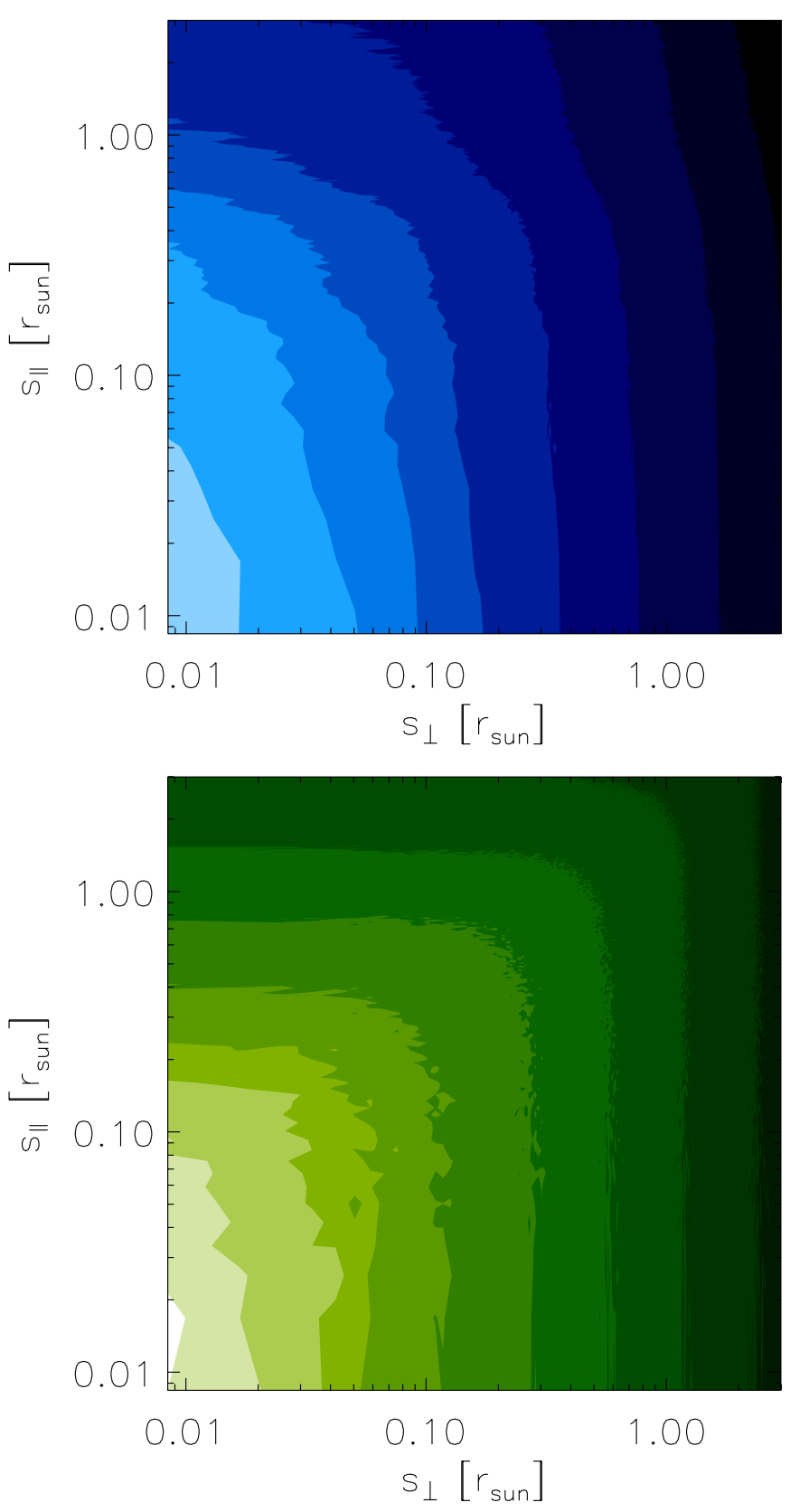

Figure 3. Two-point correlation functions for the composite model (top panel) and for the GS95 turbulence model (bottom panel).

(A color version of this figure is available in the online journal.)

turbulence spectrum used in the derivation of the critical balance by GS95 and the one used in this study. The GS95 scaling is obtained by using a continuous power-law spectrum, $k_{\perp}^{5 / 3}$, representing the Kolmogorov turbulence inertial range spectrum, while in the heliosphere the inertial range is limited in extent to only a few orders of magnitude, as the spectrum flattens at large scales into the energy-containing range. This results in flattening of the correlation function at scales of the order of $L_{c}$ and larger. Thus, when $\lambda_{\perp}<L_{\|} \lesssim L_{c}$, with $L_{\|}=L_{c}^{1 / 3} \lambda_{\perp}^{2 / 3}$, the parallel scale $L_{\|}$reaches the flattening of the correlation function before the perpendicular scale $\lambda_{\perp}$, and the ratio $L_{\|} / \lambda_{\perp}$ remains anisotropic. This range is shown by gray shading in Figure 4. As the spectrum defined by Equation (3) rolls gradually to the inertial range, it is expected to see the deviation from the GS95 form to start already at somewhat smaller scales. 


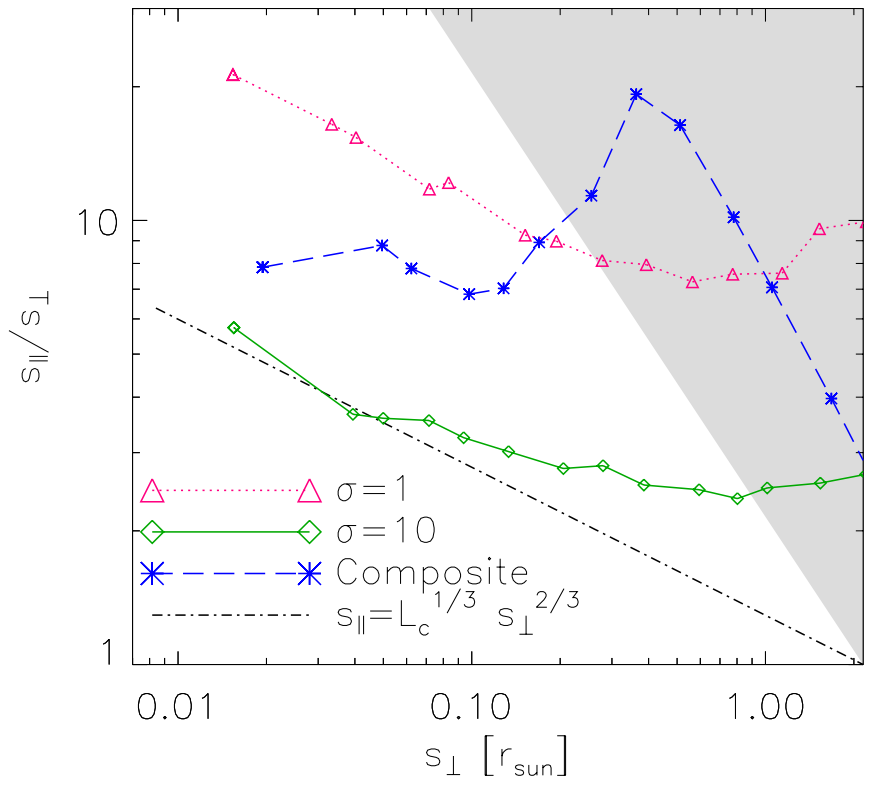

Figure 4. Scale dependence of the two models with the axis ratio $s_{\|} / s_{\perp}$. In the gray area, $L_{c}^{1 / 3} s_{\perp}^{2 / 3}$ exceeds the turnover scale $L_{c}$.

(A color version of this figure is available in the online journal.)

Solar wind turbulence observations show a somewhat smaller, but still anisotropic axis ratio of 1.5 for scales of $\sim r_{\odot}$ for slow solar wind (Dasso et al. 2005).

Thus, we conclude that our model with $\sigma=10$ displays the required scale dependence of anisotropy, with the large-scale anisotropy being consistent with the solar wind observations of Dasso et al. (2005). The gradual energy transfer model, $\sigma=1$, produces a higher level of anisotropy, exceeding the Dasso et al. (2005) result.

\subsection{Turbulence Spectrum}

In the previous section, we described the scale-dependent behavior of the two-point correlation function for different turbulence models. When studying turbulence, it is often more common to present a power spectrum, which quantifies the energy deposited in the turbulence at different scales. We present such spectra in Figure 5. The spectra are obtained from onedimensional two-point correlation functions, with the parallel spectrum defined as

$$
P_{x x}\left(k_{\|}\right)=\int C_{x x}\left(0, s_{\|}\right) e^{i k_{\|} s_{\|}} d s_{\|},
$$

and the $P_{x x}\left(k_{\perp}\right)$ similarly from $C_{x x}\left(s_{\perp}, 0\right)$. The one-dimensional spectrum can be compared to the three-dimensional spectral form given by GS95 as

$$
P(\mathbf{k})=C \frac{V_{A}^{2}}{k_{\perp}^{10 / 3} L^{1 / 3}} g\left(\frac{k_{\|} L^{1 / 3}}{k_{\perp}^{2 / 3}}\right),
$$

where $C$ is a dimensionless constant, $L$ is the isotropic excitation scale, and $g(x)$ is a function that vanishes at large $x$. From this form, the one-dimensional spectrum can be obtained using the Taylor hypothesis (Taylor 1938),

$$
P(f)=\int d^{3} \mathbf{k} P(\mathbf{k}) \delta(2 \pi f-\mathbf{k} \cdot \mathbf{V}) .
$$
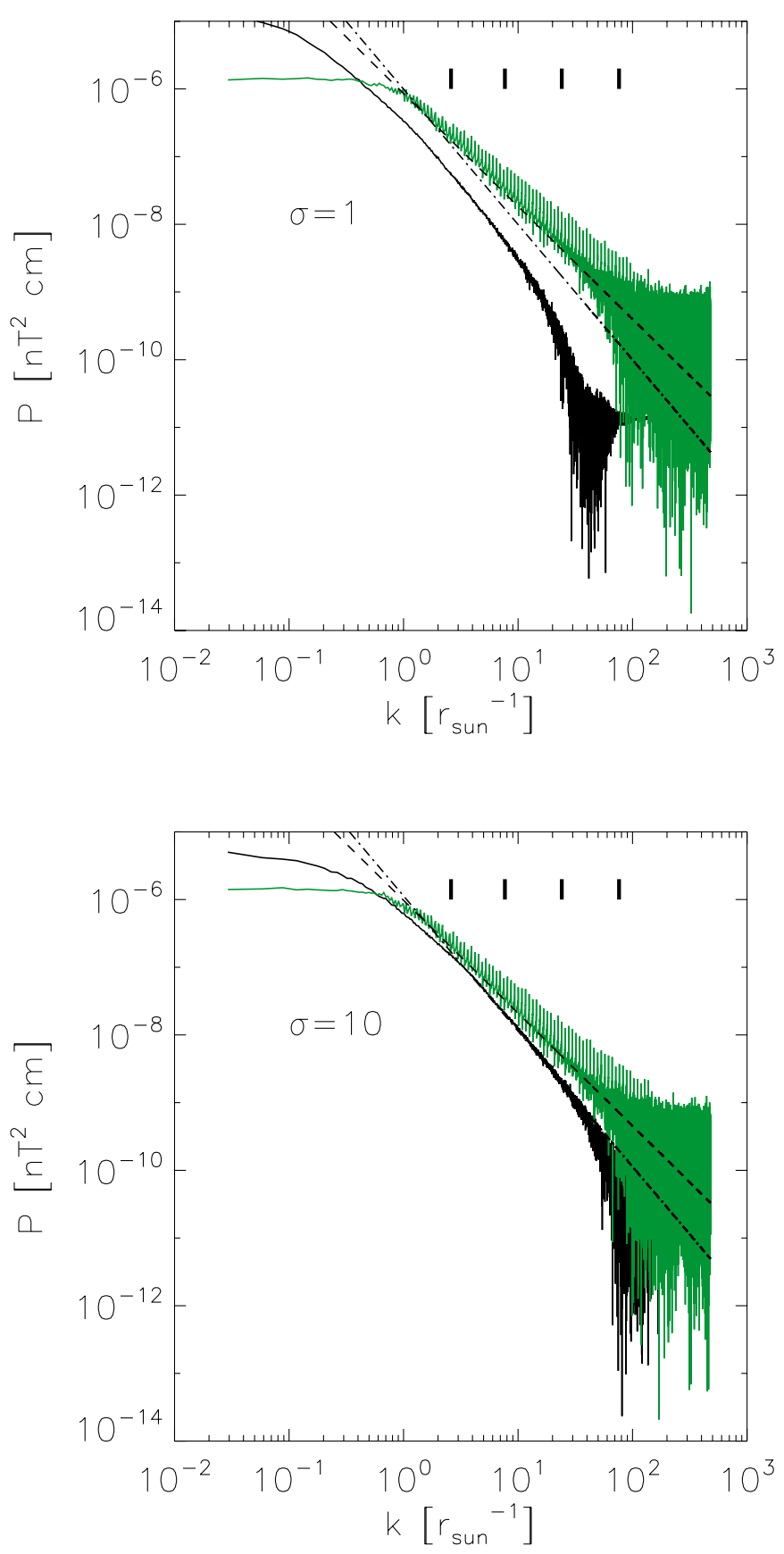

Figure 5. Parallel (black curve) and perpendicular (gray curve, green in the online version) power spectra of the GS95 turbulence model with $\sigma=1$ (top panel) and $\sigma=10$ (bottom panel). The dash-dotted and dashed curves depict the theoretical spectra given by Equations (5) and (6), respectively. The vertical bars at the top of the panels depict the inverse of the Larmor radii of protons of energies $100,10,1$, and $0.1 \mathrm{MeV}$, respectively, from left to right.

(A color version of this figure is available in the online journal.)

It is straightforward to show that the one-dimensional spectrum along the field line is proportional to $k^{-2}$ and the perpendicular spectrum to $k^{-5 / 3}$. Furthermore, we find that when using the simpler form suggested by Cho et al. (2002), $g(x)=\exp (-x)$, the spectra are given in the form

$$
P_{x x}\left(k_{\|}\right)=\frac{3}{2} C\left(\frac{1}{k_{\|} L}\right)^{2} V_{A}^{2} L
$$




$$
P_{x x}\left(k_{\perp}\right)=C\left(\frac{1}{k_{\|} L}\right)^{5 / 3} V_{A}^{2} L .
$$

We plot these spectra in Figure 5, using $L_{c}$ for the isotropic excitation scale $L$ and fitting the perpendicular spectrum, Equation (6), to the perpendicular spectrum of the simulated turbulence. It should be noted that the parallel spectrum represents a continuum instead of being composed of discrete modes, as it results from the enveloping rather than begin generated with a sum of parallel Fourier modes.

As seen in the figures, the envelope shape affects the parallel spectrum shape. For the gradual envelope profile, with $\sigma=1$ (Figure 5, top panel), the parallel spectrum has a spectral index of -2.5 , steeper and an order of magnitude below the spectrum given by Equation (5). For the steep profile, with $\sigma=10$ (Figure 5, bottom panel), the parallel spectrum has a spectral index of -2 , consistent with Equation (5), with the relative power in the parallel and perpendicular spectra consistent with Equations (5) and (6). The perpendicular spectrum is not affected by the enveloping, with the spectral index remaining at the Kolmogorov value of $-5 / 3$, the input of the model for both values of $\sigma$. The spectral indices of -2 for the parallel direction and $-5 / 3$ for the perpendicular direction were recently observed in the solar wind by Horbury et al. (2008) and Podesta (2009). The ratio $P_{\perp} / P_{\|}$in our model is 2 at $k=10 r_{\odot}^{-1}$ and 5.7 at $100 r_{\odot}^{-1}$, which is similar to the results of Horbury et al. (2008) and Podesta (2009), who find values of 1.5-5 for the range $k=10-1000 r_{\odot}^{-1}$.

\subsection{Energetic Particle Propagation}

In order to understand how the scale dependence of turbulence anisotropy affects the energetic particle propagation, we have simulated energetic protons at energies $0.1,1,10$, and $100 \mathrm{MeV}$, with the particles Larmor radius scale, $k_{L}=r_{L}^{-1}$, shown as vertical bars in Figure 5. To study the collective behavior of the particles, we have calculated the particle diffusion coefficients along and across the mean magnetic field, as described in Section 2.2, shown in Figure 6, with statistical error limits. For comparison, we have also followed particles in the composite model turbulence of Giacalone \& Jokipii (1999). The parallel diffusion coefficients for the GS95 model and the composite model are presented in the top panel of Figure 6. In the figure, the solid curve represents the composite model, whereas the dashed and dash-dotted curves (green and red in the online version) represent the GS95 model, for two values of the steepness parameter. As can be seen, the parallel diffusion coefficient is larger for the GS95 model and depends strongly on the steepness parameter of the scale-dependent enveloping. This can be understood on the basis of lower energy in parallel fluctuations to scatter the particles in the $\sigma=1$ case compared to the $\sigma=10$ case. The dependence of the parallel diffusion coefficient on particle energy in the GS95 model also differs from the composite model, with the diffusion coefficient decreasing slower at decreasing energies. This can be understood as the effect of the steeper spectrum, which results in a smaller relative energy in the small-scale fluctuations that scatter the lower energy particles.

We also present theoretical estimations of the parallel diffusion coefficient in Figure 6. The dotted line represents the QLT $\kappa_{\|}$, as given by Giacalone \& Jokipii (1999) presented in their work for comparison with the composite and isotropic turbulence models. Their estimation is based on an equation that is
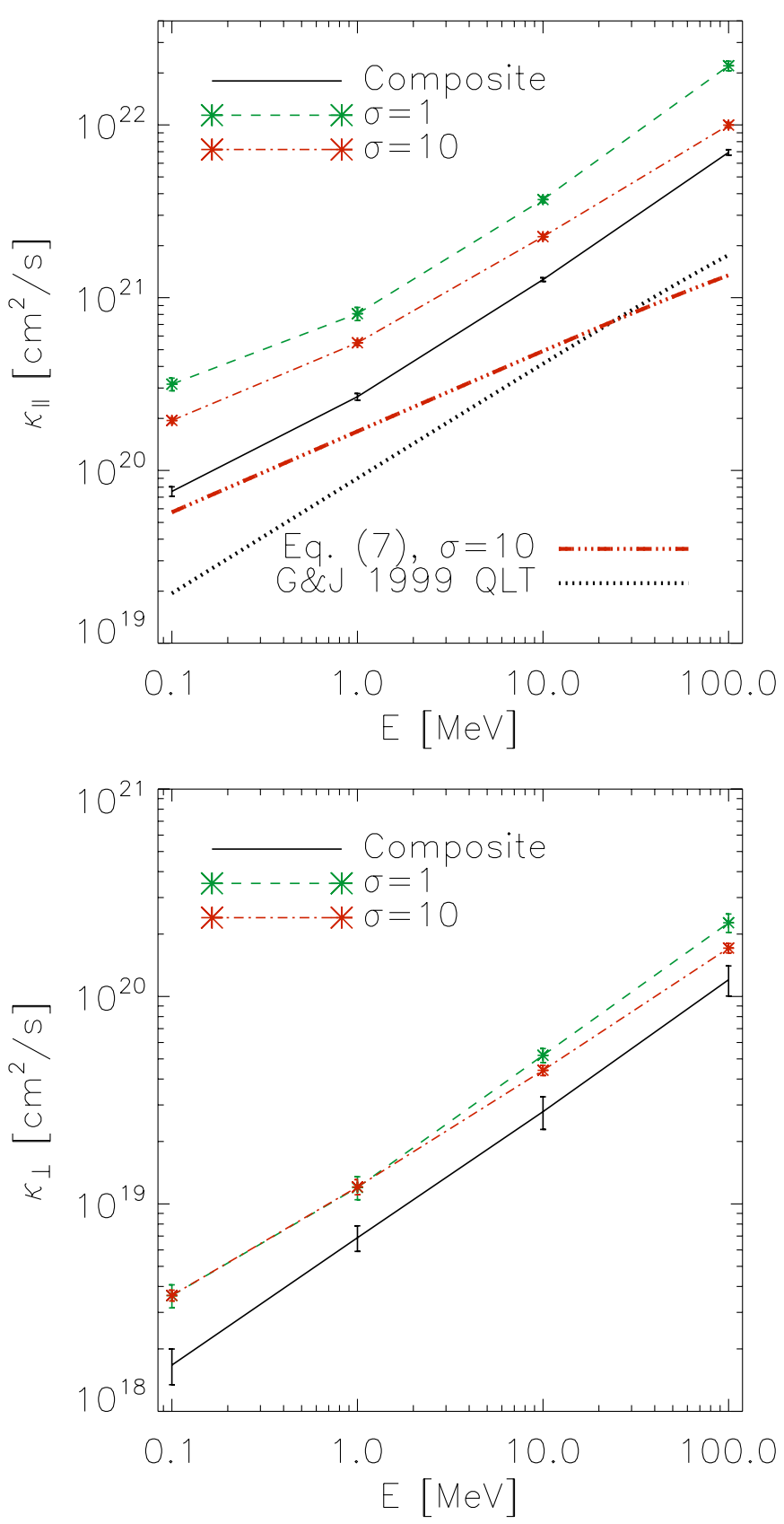

Figure 6. Parallel (top panel) and perpendicular (bottom panel) energetic particle diffusion coefficients in composite turbulence (solid curve), and in the GS95 turbulence (dashed and dash-dotted curves, green and red in the online version), as a function of proton energy, for turbulence amplitude $B_{1} / B_{0}=1$. In the top panel, the dashed curve depicts the QLT diffusion coefficient for the composite model, as given by Giacalone \& Jokipii (1999), and the tripledot-dash line Equation (7) for the $\sigma=10$ case.

(A color version of this figure is available in the online journal.)

valid only for parallel Alfvén waves (the slab waves). It should be noted that in a composite turbulence, the two-dimensional component does not have a significant contribution to parallel scattering (e.g., Bieber et al. 1994). If we assume that only the slab component contributes to the parallel scattering, then the 80\%:20\% mix of two-dimensional and slab modes implies a factor five rise of the dotted curve in the upper panel of Figure 6, which fits the simulated composite model results well. 
Comparison of the theoretical parallel diffusion coefficient with our results for the GS95 turbulence is not straightforward, as the QLT for magnetostatic turbulence results in an infinite diffusion coefficient for parallel spectrum $\propto k_{\|}^{-2}$ and steeper. This resonance gap problem, due to the lack of scattering at low particle pitch angle cosines, $\mu=v_{\|} / v$, has been studied in the scientific literature, and several mechanisms for its closure have been suggested (e.g., Schlickeiser \& Achatz 1993; Bieber et al. 1994; Ng \& Reames 1995; Vainio 2000). As shown by, e.g., Vainio (2000), if scattering at low pitch angles after the closure of the resonance gap is weak, then the diffusion coefficient is dominated by this low rate of scattering, and thus by the closure mechanism. If, however, the resonance gap is efficiently closed, then the pitch angle diffusion coefficient can be estimated by

$$
\kappa_{\|} \propto v r_{L} \frac{B_{0}^{2}}{r_{L}^{-1} P\left(r_{L}^{-1}\right)} h\left(\mu_{g}\right),
$$

where $r_{L}$ is the particle's Larmor radius and $h\left(\mu_{g}\right)$ is a dimensionless function that depends on the spectral index of the parallel turbulence and the pitch angle $\mu_{g}$ below which the resonance gap closure mechanisms overcome the QLT result. This form is valid only for a power-law spectrum of the turbulence, and thus the higher energies in this study are expected to have larger diffusion coefficients, compared to the estimation. In addition, it should be noted that the above considerations are valid for slab turbulence. As our model contains a wealth of oblique wave modes, implied by the correlation function, the QLT would give a wealth of harmonic resonances in addition to the resonance at inverse Larmor radius.

We present the theoretical parallel diffusion coefficient, as given by Equation (7), in Figure 6, with a tripledot-dash line, for our model with steepness parameter $\sigma=10$. The power spectrum in Equation (7) is obtained as a power-law fit to the parallel spectrum (Figure 5). The exact scaling, given by $h\left(\mu_{g}\right)$, is not known, and thus we can only compare the trends of the diffusion coefficients. As can be seen, the QLT trend tracks the GS95 model only at low energies. This suggests that the resonance gap effects must be taken into account when estimating the mean free path at steep spectra.

The perpendicular diffusion coefficients are shown in the bottom panel of Figure 6 . The perpendicular diffusion is stronger in the GS95 turbulence, by a factor of two, as compared to the composite model. The dependence on the enveloping shape is small except for the highest energies used in the study. Thus, the perpendicular and parallel diffusion coefficients behave differently with respect to the exact description of the critically balanced turbulence.

\section{DISCUSSION AND CONCLUSIONS}

In this work, we study how energetic particles propagate in critically balanced turbulence. We build turbulence with scaledependent anisotropy resulting from critical balance by using envelopes of a length that follows the required scaling between the perpendicular wavenumber and the parallel variation scale. Analysis of the 2-point correlation function and the spectra shows that for the envelope steepness parameter $\sigma=10$, our model is consistent with the GS95 critical balance scaling and the observations of solar wind turbulence (Dasso et al. 2005; Horbury et al. 2008; Podesta 2009). The gradual shape, $\sigma=1$ (see Figure 2), results in more pronounced anisotropy. The case of $\sigma=10$, however, can be considered more realistic, as the nonlinear interactions between the waves depend on their relative phases (e.g., Luo \& Melrose 2006), resulting in an uneven evolution of the wave packets. The structure of rapid change after an extended period of unchanged turbulence can also be viewed as intermittent turbulence. We note, however, that in our model, the probability distribution of magnetic field increments $\Delta B_{x}\left(s_{\perp}, s_{\|}\right)=B_{x}\left(r_{\perp}+s_{\perp}, r_{\|}+s_{\|}\right)-$ $B_{x}\left(r_{\perp}, r_{\|}\right)$does not significantly deviate from Gaussian, unlike for the observed and modeled turbulence (e.g., Greco et al. 2009).

The scale dependence of the turbulence results in a notable difference in the power spectra along and across the mean magnetic field, as shown by Figure 5. Thus, it is expected that the energetic particle transport differs from the composite model of Giacalone \& Jokipii (1999), where the spectral indices are both equal to $5 / 3$. Our simulations show that the effect for the parallel diffusion coefficient is significant, with a 1.5-2.5-fold increase, depending on the particle energy, for $\sigma=10$ (top panel of Figure 6). An increase of the diffusion coefficient with a steepening parallel spectrum is consistent with the standard quasilinear theory (e.g., Dung \& Schlickeiser 1990). Our analysis shows, however, that the simple estimate based on QLT, for the parallel wave modes (Equation (7)), fails to describe the parallel diffusion coefficient at higher energies.

The perpendicular propagation is also affected by the scale dependence of the turbulence, with the perpendicular diffusion coefficient being larger by a factor of two compared to the one obtained with the composite model. This can be understood on the basis of the work of Matthaeus et al. (2003). In their formulation, the perpendicular diffusion coefficient depends on the parallel diffusion coefficient. Thus, a different form for the parallel component spectrum would influence the cross-field transport of the energetic particles. Shalchi et al. (2010) used the further-developed model by Shalchi (2010) to calculate the perpendicular diffusion coefficient in a GS95-type spectrum. Their $\kappa_{\perp} / \kappa_{\|}$decreases $\sim 5$-fold compared to the composite model, and deviates from our result, where the ratio does not change for $\sigma=10$. However, in their work, they did not estimate the parallel diffusion coefficient consistently using the GS95-spectrum, but used standard quasilinear theory (Jokipii 1966) with the Kolmogorov spectrum and a model based on Galactic cosmic-ray observations. Thus, their results are not comparable to $\kappa_{\perp} / \kappa_{\|}$in our model. The insensitivity of $\kappa_{\perp}$ to the parallel diffusion coefficient in their model is in line with our results of insensitivity of $\kappa_{\perp}$ to the changes in the turbulence caused by the change of the envelope shape. This insensitivity can also be implied from the theoretical work by Matthaeus et al. (2003, their Equation (7)), where the rapidly decreasing power spectrum may diminish the link between the parallel and perpendicular diffusion.

Determining the correct scattering parameters in the interplanetary space is very important for the analysis of SEP events. Studies have shown that the scattering along the interplanetary magnetic field can significantly affect the accuracy of the SEP event onset analysis at 1 AU (Lintunen \& Vainio 2004; Sáiz et al. 2005; Laitinen et al. 2010). Recently, Giacalone \& Jokipii (2012) have shown that varying the diffusion coefficients and the ratio of the perpendicular and parallel diffusion coefficients in the simulations can result in a wide variety of intensity profiles at $1 \mathrm{AU}$ for an impulsive SEP event. Therefore, the two-fold increase in the parallel and perpendicular transport coefficients reported in this paper will significantly influence the characteristics of SEP events. 
We acknowledge support from the UK Science and Technology Facilities Council (STFC) (grant ST/J001341/1) and from the European Commission FP7 Project COMESEP (263252). J.K. acknowledges STFC support via PhD studentship. Access to the University of Central Lancashire's High Performance Computing Facility is gratefully acknowledged.

\section{REFERENCES}

Aschwanden, M. 2012, SSRv, 171, 3

Beresnyak, A., Yan, H., \& Lazarian, A. 2011, ApJ, 728, 60

Bieber, J. W., Matthaeus, W. H., Smith, C. W., et al. 1994, ApJ, 420, 294

Bieber, J. W., Wanner, W., \& Matthaeus, W. H. 1996, JGR, 101, 2511

Cane, H. V., Richardson, I. G., \& von Rosenvinge, T. T. 2010, JGRA, 115, 8101

Cho, J., Lazarian, A., \& Vishniac, E. T. 2002, ApJ, 564, 291

Dalla, S., Balogh, A., Krucker, S., et al. 2003, AnGeo, 21, 1367

Dalla, S., \& Browning, P. K. 2005, A\&A, 436, 1103

Dasso, S., Milano, L. J., Matthaeus, W. H., \& Smith, C. W. 2005, ApJL, 635, L181

Dresing, N., Gómez-Herrero, R., Klassen, A., et al. 2012, SoPh, 281, 281

Dung, R., \& Schlickeiser, R. 1990, A\&A, 237, 504

Giacalone, J., \& Jokipii, J. R. 1999, ApJ, 520, 204

Giacalone, J., \& Jokipii, J. R. 2012, ApJL, 751, L33

Goldreich, P., \& Sridhar, S. 1995, ApJ, 438, 763

Gopalswamy, N., Xie, H., Yashiro, S., et al. 2012, SSRv, 171, 23

Greco, A., Matthaeus, W. H., Servidio, S., Chuychai, P., \& Dmitruk, P. 2009, ApJL, 691, L111
Horbury, T. S., Forman, M., \& Oughton, S. 2008, PhRvL, 101, 175005

Jokipii, J. R. 1966, ApJ, 146, 480

Laitinen, T., Dalla, S., \& Kelly, J. 2012, ApJ, 749, 103

Laitinen, T., Huttunen-Heikinmaa, K., \& Valtonen, E. 2010, in AIP Conf. Proc., Vol. 1216, Twelfth Int. Solar Wind Conf. (Melville, NY: AIP), 249

Lintunen, J., \& Vainio, R. 2004, A\&A, 420, 343

Liu, Y., Luhmann, J. G., Bale, S. D., \& Lin, R. P. 2011, ApJ, 734, 84

Luo, Q., \& Melrose, D. 2006, MNRAS, 368, 1151

Matthaeus, W. H., Qin, G., Bieber, J. W., \& Zank, G. P. 2003, ApJL, 590, L53

Ng, C. K., \& Reames, D. V. 1995, ApJ, 453, 890

Parker, E. N. 1965, P\&SS, 13, 9

Podesta, J. J. 2009, ApJ, 698, 986

Press, W. H., Teukolsky, S. A., Vetteling, W. T., \& Flannery, B. P. 1993, Numerical Recipes in Fortran: The Art of Scientific Computing (2nd ed.; New York: Cambridge Univ. Press)

Qin, G. 2002, PhD thesis, Univ. of Delaware

Qin, G., Matthaeus, W. H., \& Bieber, J. W. 2002, ApJL, 578, L117

Ruffolo, D., Chuychai, P., Wongpan, P., et al. 2008, ApJ, 686, 1231

Sáiz, A., Evenson, P., Ruffolo, D., \& Bieber, J. W. 2005, ApJ, 626, 1131

Schlickeiser, R., \& Achatz, U. 1993, JPIPh, 49, 63

Shalchi, A. 2010, ApJL, 720, L127

Shalchi, A., Büsching, I., Lazarian, A., \& Schlickeiser, R. 2010, ApJ, 725, 2117

Shebalin, J. V., Matthaeus, W. H., \& Montgomery, D. 1983, JPIPh, 29, 525

Taylor, G. I. 1938, RSPSA, 164, 476

Tu, C.-Y., \& Marsch, E. 1995, SSRv, 73, 1

Vainio, R. 2000, ApJS, 131, 519

Wisniewski, M., Spanier, F., \& Kissmann, R. 2012, ApJ, 750, 150

Zimbardo, G., Pommois, P., \& Veltri, P. 2006, ApJL, 639, L91 